\title{
Method of Seismic Tsunami Hazard Estimate Effect Coupling Uncertainty of Magnitude and Focal Depth
}

\author{
Zhe Liu, Jianwei Tian, Luchuan Ren* \\ Institute of Disaster Prevention, China Earthquake Administration, Beijing East Yanjiao, 101601
}

\section{耦合震级和震源深度不确定性效应的地震海啸 危险性估计方法 \\ 刘哲, 田建伟, 任鲁川 \\ 中国地震局防灾科技学院, 北京东燕郊 101601}

\begin{abstract}
:
We discuss a method to estimate the seismic tsunami hazard coupling uncertainty of upper magnitude limit and focal depth in this paper. The method of estimating uncertainties of upper magnitude limit and focal depth is discussed. Manila trench and Ryukyu trench subduction zones are chosen to be the potential seismic tsunami source areas and five off-shore sites near to Chinese southeast coastal region are used as site-specific. The uncertainty of upper magnitude limit is estimated by generalized extreme value method and the uncertainty of focal depth is calculated by probabilistic statistics method. Under the assumption that earthquake with the upper magnitude limit occurred in Manila trench and Ryukyu trench subduction zone, the seismic tsunami hazards that exceed $0.5 \mathrm{~m}$ of tsunami wave height at five site-specific are estimated.
\end{abstract}

Keywords: Seismic tsunami; Hazard estimation; Uncertainty; Upper magnitude limit; Focal depth

作者简介: 刘哲 (1991-), 男, 硕士研究生, 主要从事地震灾害预测研究 e-mail:

kuailexingyu@sina.com

通讯作者: 任鲁川（1958-）, 男, 博士, 教 授, 主要从事灾害风险分析方面研究 $e^{-}$-mai1: ren1uchuan@sina.com
摘 要

探讨耦合震级上限和震源深度不确定性效应 的地震海啸危险性估计方法, 论述了震级上限 和震源深度不确定性的估计方法。选取中国东 南沿海地区五个近岸特定场点, 以马尼拉海沟 俯冲带和琉球海沟俯冲带作为潜在地震海啸 源区, 采用广义极值模型估计震级上限并分析 其不确定性特征, 通过统计震源深度的优势分 布, 耦合震级上限和震源深度不确定性效应, 估计马尼拉海沟俯冲带和琉球海沟俯冲带发 生可能最大地震时五个特定场点海啸波高超 过 $0.5 \mathrm{~m}$ 的地震海啸危险性。

关键词: 地震海啸; 危险性估计; 不确定性;震级 上限;震源深度

1 引言

震级是地震释放能量大小的标度, 震级上 限的估计是地震危险性分析的重要环节之一。 李伟等[1]假设冲绳海槽发生 9.0 级特大地震, 考虑了其 35 秒破裂时间, 对海啸波在中国东 南沿海浅水大陆架的传播过程进行研究。温燕 林等[2]将琉球海沟与日本海沟、智利海沟等一 些发生过 9.0 级以上地震的海沟进行地质构造 类比, 设定琉球海沟俯冲带发生 9.0 级特大地 震的情景, 评估了中国东南沿海地区的海啸风 险。张琨等[3]通过分析琉球海沟俯冲带的地震 活动性, 利用广义极值模型, 得到琉球海沟俯 冲带震级上限的估计值及其置信区间。在地震 


\section{Risk Analysis and Crisis Response in Big Data Era (RAC-16)}

危险性分析中, 很多学者[4]-[7]通过估计潜在 震源区 $\mathrm{b}$ 值和年发生率 $\mathrm{V}$ 的不确定性, 利用 逻辑树的方法, 对特定场点的地震危险性进行 评价。但在估计地震海啸危险性时，还需考虑 断层错动对海水运动的效应及海底地形对海 啸波传播的影响。为考虑些影响, 目前大多数 学者 $[8,9]$ 都利用海啸数值模拟进行地震海啸 危险性分析, 并对这些海啸数值模式的可靠性 进行了检验。但在海啸数值模拟时, 仍需考虑 潜在地震海啸源区潜源参数的不确定性效应。 温瑞智等 [10]指出, 估计特定场点的地震海啸 危险性时, 需考虑震级的不确定性效应。任鲁 川等和 ERIC 等 $[11,12]$ 总结了耦合潜源参数不 确定性效应的地震海啸危险性估计的一般原 理与方法。提到在估计特点场点的地震海啸危 险性时, 可将潜源参数 (断层的长度、宽度、 走向角、倾角、滑动角, 震级, 震中的经度、 纬度, 震源深度) 的不确定性效应进行耦合, 并通过海啸数值模拟得到特定场点的海啸波 高, 来估计特定场点的地震海啸危险性。本文 将对耦合震级上限和震源深度不确定性效应 的地震海啸危险性估计方法进行探讨, 并以马 尼拉海沟和琉球海沟俯冲带作为潜在地震海 啸源区, 估计中国东南沿海地区五个特定场点 的地震海啸危险性。

\section{2.研究方法与实施步骤}

耦合潜源参数不确定性效应估计地震海 啸危险性可分为六步。第一步, 选取沿海地区 特定场点; 第二步, 界定潜在地震海啸源区; 第三步, 为潜源参数赋值; 第四步, 海啸数值 模拟; 第五步, 耦合震级上限和震源深度的不 确定性效应; 第六步, 估计特定场点的地震海 啸危险性。具体研究方法可详见文献 11。

在估计特定场点的地震海啸危险性时, 假 设特定场点遭受地震海啸灾害袭击的事件为 泊松过程, 可用下式估计特定场点的地震海啸 危险性

$$
P\left(r, T, h_{c r i t}\right)=1-e^{-N\left(r, h_{\text {crit }}\right) T}
$$

式中, $P\left(r, T, h_{c r i t}\right)$ 表示未来 $T$ 年内, 特定场 点遭受超过特定海啸波高的地震海啸危险性; $h_{c r i t}$ 表示特定场点的临界海啸波高; $r$ 表示可
能对特定场点造成地震海啸灾害的潜在地震 海啸源; $N\left(r, h_{c r i t}\right)$ 表示特定场点地震海啸年 发生率。由于耦合的潜源参数中包含震级上 限，其时间尺度趋于无穷大。因此，计算得到 的地震海啸发生率就是特定场点未来遭受最 大震级时的发生率, 因此需将公式 1-1 调整为:

$$
P\left(r, T, h_{c r i t}\right)=1-e^{-N\left(r, h_{\text {crit }}\right)}
$$

\section{3.基于广义极值模型的震级上限估计不确定 性研究}

广义极值模型是描述研究对象尾部特征 的一种概率模型, 张锟等 [3]和钱小仕等[13]曾 将其用来估计震级上限的不确定性。假设

$X_{1}, X_{2}, \cdots, X_{n}$ 为每一时段内的最大地震震

级数据, 并且它们相互独立同分布, 其分布函 数可以用统一形式表示为:

$$
F(x)=\exp \left[-\left(1+\xi \frac{x-\mu}{\sigma}\right)^{-1 / \xi}\right]
$$

式中, $\xi$ 为形状参数, $\mu$ 为位置参数, $\sigma$ 为 尺度参数。当 $\xi<0$ 时, 震级服从 Weibull 分 布，震级上限的极大似然估计值：

$$
z_{0}=\mu-\frac{\sigma}{\xi}
$$

其方差可表示为

$$
\operatorname{Var}\left(z_{0}\right) \approx \nabla z_{0}{ }^{T} V \nabla z_{0} \quad(3-3)
$$

其中, $z_{0}$ 代表潜在地震海啸源区的震级上限, $V$ 代表广义极值模型中三个参数的协方差矩 阵, $\nabla z_{0}$ 代表震级上限 $z_{0}$ 在广义极值模型三 个参数处的偏导数矩阵, $\nabla z_{0}{ }^{T}$ 是 $\nabla z_{0}$ 的转置 矩阵。由此可分析震级上限估计的不确定性特 征。

\section{4.估计中国东南沿海地区特定场点的地震海 啸危险性}

本文将中国东南沿海地区五个近岸场点 
Risk Analysis and Crisis Response in Big Data Era (RAC-16)

作为特定场点（表 1)。

表 1 中国东南沿海特定场点

\begin{tabular}{cccccc}
\hline & $\begin{array}{c}\text { 特定 } \\
\text { 场点 } \mathbf{1}\end{array}$ & $\begin{array}{c}\text { 特定 } \\
\text { 场点 } \mathbf{2}\end{array}$ & $\begin{array}{c}\text { 特定 } \\
\text { 场点 } \mathbf{3}\end{array}$ & $\begin{array}{c}\text { 特定 } \\
\text { 场点 } \mathbf{4}\end{array}$ & $\begin{array}{c}\text { 特定 } \\
\text { 场点 } \mathbf{5}\end{array}$ \\
\hline $\begin{array}{c}\text { 纬度 } \\
\left({ }^{\circ}\right)\end{array}$ & 21 & 23 & 25 & 27.0 & 29.0 \\
经度 & & & & & \\
$\left({ }^{\circ}\right)$ & 112.3 & 119.2 & 119.7 & 119.2 & 122.6 \\
水深 \\
$(\mathrm{m})$
\end{tabular}

本文选取马尼拉海沟和琉球海沟俯冲带 作为潜在地震海啸源区, 通过检索美国地质调 查局网站(www.usgs.gov)1900-2010 年 Mw6 以 上潜在地震海啸源区的地震目录, 绘制震中分 布图（图 1）。

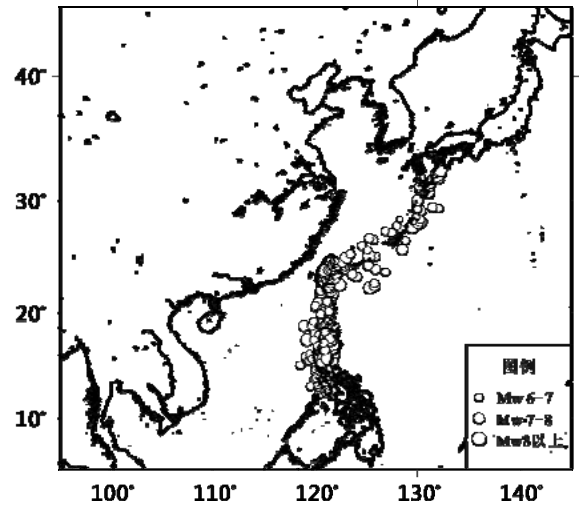

图 1 潜源区及其邻域的震中分布图

图 1 中, 显示出马尼拉海沟和琉球海沟俯 冲带的地震分布存在空间对称现象，与延军平 等[14]探讨的部分地区地震存在空间对称轴的 结论一致。

\section{1 估计潜源区震级上限和震源深度的不确} 定性

4.1.1 潜源区震级上限估计的不确定性

限于篇幅, 本文只给出马尼拉海沟俯冲带 震级上限估计值不确定性的估计过程。以 5 年 为一时间时段, 对各个时间段的最大震级数据 进行广义极值分布模型拟合, 得到三个参数的 极大似然估计值及其标准差（表 2），并对广 义极值模型拟合状况进行诊断（图 2)。图 $2 a$
为概率图, 图 $2 \mathrm{~b}$ 为分位数图, 图 $2 \mathrm{c}$ 为重现水 平图, 图 $2 \mathrm{~d}$ 为概率密度图。概率图、分位数 图和概率密度图均显示震级数据的累计概率、 分位数和概率密度与模型估计的近似相同, 重 现水平图显示震级数据的分位数均落在模型 估计的分位数的置信区间内。由上述四个诊断 图表明, 广义极值模型拟合的状况良好。将广 义极值模型三个参数的估计值代入公式 3-2 和 3-3 中, 得到马尼拉海沟俯冲带震级上限的估 计值及其方差。

表 2 马尼拉海沟俯冲带广义极值分布三个参 数的极大似然对数估计值及其标准差

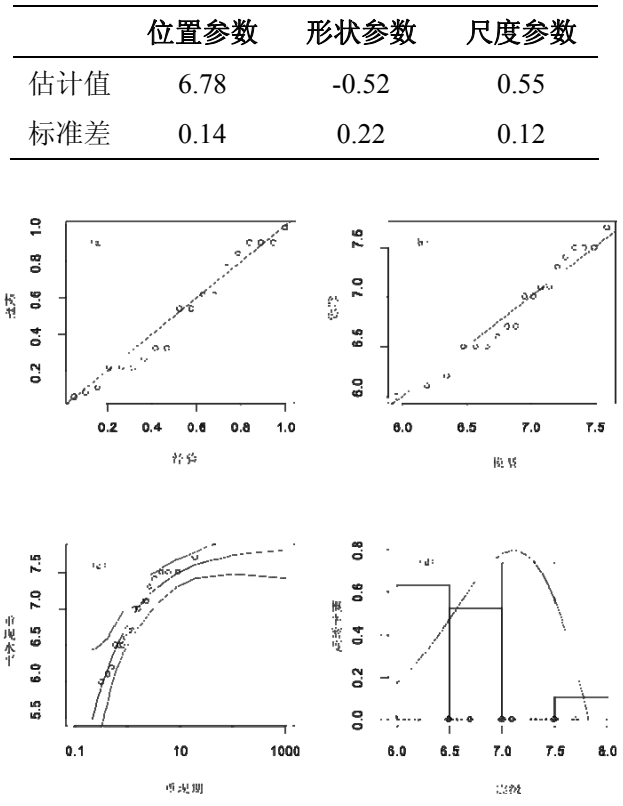

图 2 广义极值模型拟合最大震级数据的诊断图 (a)概率图 (b)分位数图 (c)重现水平图 (d) 概 率密度图

同样地, 也可得到琉球海沟震级上限的估计值 及其方差（表 3）。

表 3 潜源区震级上限估计值及其方差

\begin{tabular}{ccc}
\hline & $\begin{array}{c}\text { 马尼拉海沟 } \\
\text { 沉降带 }\end{array}$ & $\begin{array}{c}\text { 琉球海沟 } \\
\text { 沉降带 }\end{array}$ \\
\hline 震级上限估计值 $(\mathrm{Mw})$ & 7.9 & 8.5 \\
方差 & 0.1 & 0.7 \\
\hline
\end{tabular}




\section{Risk Analysis and Crisis Response in Big Data Era (RAC-16)}

4.1.2 估计潜源区震源深度的不确定性

利用检索的地震目录, 可分析马尼拉海沟 和琉球海沟俯冲带潜在地震海啸源区震源深 度的分布频率（图 3)，及其优势分布。本文 按照震源深度优势分布进行等比例重新分配

(表 4)。
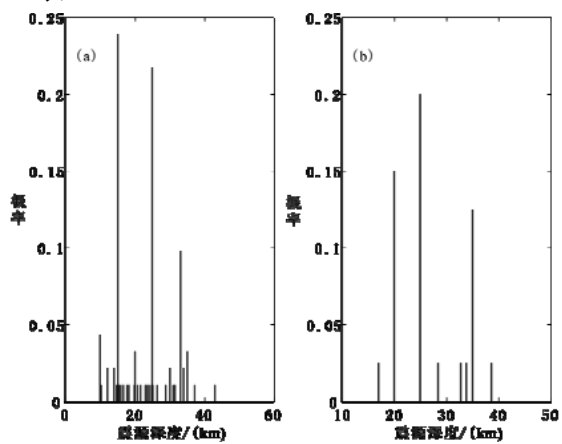

图 3 潜源区震源深度分布频率

(a) 马尼拉海沟俯冲带 (b)琉球海沟俯冲带

表 4 潜源区震源深度优势分布及其频率

\begin{tabular}{ccccc}
\hline & $\begin{array}{c}\text { 马尼拉海沟沉 } \\
\text { 降带 }\end{array}$ & \multicolumn{2}{c}{$\begin{array}{c}\text { 琉球海沟沉 } \\
\text { 降带 }\end{array}$} \\
\hline 震源深 & 15 & 25 & 20 & 25 \\
度 $(\mathrm{km})$ & & & & \\
频率 & 0.24 & 0.23 & 0.15 & 0.20 \\
逻辑树 & 0.51 & 0.49 & 0.43 & 0.57 \\
\hline
\end{tabular}

\section{2 海啸数值模拟}

\subsection{1 潜源参数赋值}

基于潜源区震级上限估计值的不确定性, 采用蒙特卡洛方法进行采样, 并配合震源深度 的优势分布, 得到潜源区震级与震源深度的海 啸数值模拟的样本（表 5 表 6）。

\begin{tabular}{ccccc} 
表 5 & \multicolumn{4}{c}{ 马尼拉海沟俯冲带震级与震源深度 } \\
\hline 序 & 震级 & 震源深 & 震级 & 震源深 \\
号 & $(\mathrm{Mw})$ & 度 $(\mathrm{km})$ & $(\mathrm{Mw})$ & 度 $(\mathrm{km})$ \\
\hline 1 & 7.9 & 15 & 7.9 & 25 \\
2 & 7.7 & 15 & 7.7 & 25 \\
3 & 7.9 & 15 & 7.9 & 25 \\
4 & 8.0 & 15 & 8.0 & 25 \\
5 & 7.8 & 15 & 7.8 & 25 \\
6 & 8.0 & 15 & 8.0 & 25 \\
7 & 7.8 & 15 & 7.8 & 25 \\
8 & 8.1 & 15 & 8.1 & 25 \\
9 & 7.9 & 15 & 7.9 & 25 \\
\hline
\end{tabular}

表 6 琉球海沟俯冲带震级与震源深度

\begin{tabular}{ccccc}
\hline $\begin{array}{c}\text { 序 } \\
\text { 号 }\end{array}$ & $\begin{array}{c}\text { 震级 } \\
(\mathrm{Mw})\end{array}$ & $\begin{array}{c}\text { 震源深 } \\
\text { 度 }(\mathrm{km})\end{array}$ & $\begin{array}{c}\text { 震级 } \\
(\mathrm{Mw})\end{array}$ & $\begin{array}{c}\text { 震源深 } \\
\text { 度 }(\mathrm{km})\end{array}$ \\
\hline 1 & 8.7 & 20 & 8.7 & 25 \\
2 & 8.0 & 20 & 8.0 & 25 \\
3 & 8.5 & 20 & 8.5 & 25 \\
4 & 8.4 & 20 & 8.4 & 25 \\
5 & 9.1 & 20 & 9.1 & 25 \\
6 & 8.0 & 20 & 8.0 & 25 \\
7 & 8.9 & 20 & 8.9 & 25 \\
8 & 8.3 & 20 & 8.3 & 25 \\
9 & 8.6 & 20 & 8.6 & 25
\end{tabular}

Xiaoming Wang 等[15]统计得到马尼拉海沟俯 冲带震中位置的走向 $34^{\circ}$, 倾角 $20^{\circ}$, 断层长 $200 \mathrm{~km}$, 断层宽 $20 \mathrm{~km}$; 琉球海沟俯冲带震中 位置的走向 $247^{\circ}$, 倾角 $16^{\circ}$ 断层长 $730 \mathrm{~km}$, 断 层宽 $20 \mathrm{~km}$ 。地震的滑动角均设为 $90^{\circ}$ 。潜源参 数中的震中位置参数, 可依据地震空区和特征 地震进行选取, 选取马尼拉海沟俯冲带的 $18.7^{\circ} \mathrm{N}, 119.8^{\circ} \mathrm{E}$ 和琉球海沟 $23.4^{\circ} \mathrm{N}, 124.1^{\circ} \mathrm{E}$ 作 为震中位置。由此, 可采用逻辑树模型和概率 统计模型组成地震海啸危险性估计方案 (图 4) 。

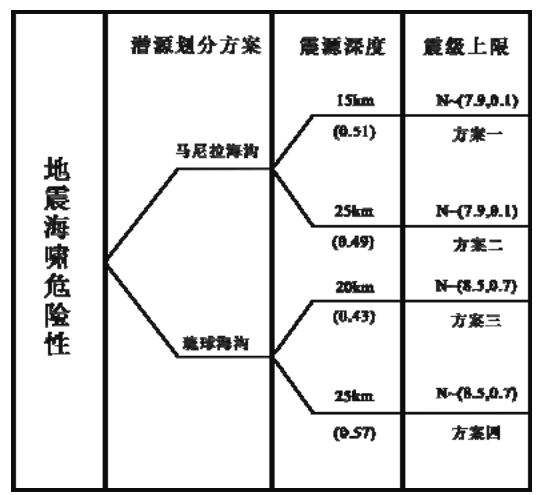

图 4 地震海啸危险性估计方案

\subsection{2 海啸数值模拟}

本文采用 COMCOT 海啸数值模式, 对研究地 区进行海啸数值模拟。嵌套模式采用两层网格 嵌套。其中, 第一层网格嵌套区域为 $10^{\circ}-40^{\circ} \mathrm{N}$, $105^{\circ}-135^{\circ} \mathrm{E}$, 时间步长为 $5^{\prime}$; 第二层网格嵌套 区域分别为 $25^{\circ}-30^{\circ} \mathrm{N}, 118^{\circ}-123^{\circ} \mathrm{E} ; 20^{\circ}-25^{\circ} \mathrm{N}$, $115^{\circ}-120^{\circ} \mathrm{E} ; 18^{\circ}-23^{\circ} \mathrm{N}, 110^{\circ}-115^{\circ} \mathrm{E}$ (图 5)。 
Risk Analysis and Crisis Response in Big Data Era (RAC-16)

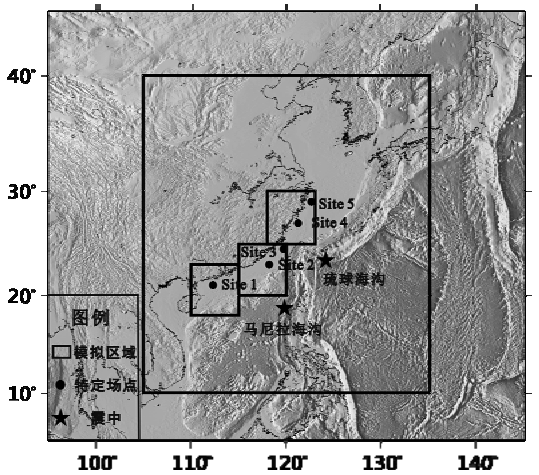

图 5 海啸数值模拟示意图

地形数据选用美国地质调查局(USGS)的 etopo1 数据。限于篇幅, 本文只给出方案一中 第 8 组潜源参数对应特定场点 $1\left(21.0^{\circ} \mathrm{N}\right.$, $112.3^{\circ} \mathrm{E}$ ) 的海啸波高随时间变化曲线 (图 6)。 从上述海啸波高数据中, 篮选出最大海啸波高 为 $0.60 \mathrm{~m}$ 。以此类推, 得到该特定场点的所有 最大海啸波高数据（表 7）。

表 7 中国东南沿海地区特定场点 1 的最大海 啸波高(单位: m)

\begin{tabular}{ccccc}
\hline 序号 & 方案一 & 方案二 & 方案三 & 方案四 \\
\hline 1 & 0.46 & 0.42 & 0.57 & 0.59 \\
2 & 0.45 & 0.39 & 0.40 & 0.42 \\
3 & 0.46 & 0.42 & 0.44 & 0.42 \\
4 & 0.54 & 0.52 & 0.43 & 0.41 \\
5 & 0.45 & 0.40 & 1.53 & 1.55 \\
6 & 0.54 & 0.52 & 0.40 & 0.42 \\
7 & 0.45 & 0.40 & 0.79 & 0.60 \\
8 & 0.60 & 0.53 & 0.42 & 0.40 \\
9 & 0.46 & 0.42 & 0.49 & 0.48 \\
10 & 0.45 & 0.40 & 0.42 & 0.40 \\
\hline
\end{tabular}

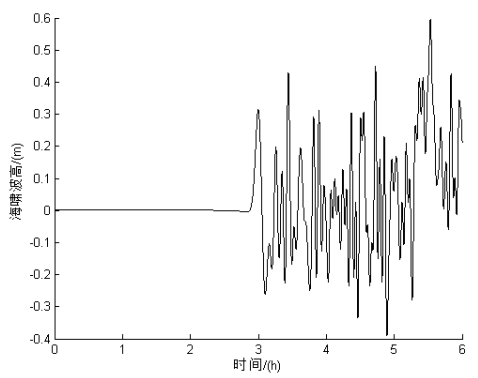

图 6 特定场点 1 波高随时间的变化

\section{3 估计特定场点的地震海啸危险性}

假设最大海啸波高数据服从正态分布, 则 方案一对应的最大海啸波高分布形式为

$(\mathrm{N} \sim(0.49,0.05))$, 选取临界海啸波高为 0.5 米

时, $m_{\text {crit }}=7.9$, 经计算得到超越临界海啸波 高的概率为 $\phi\left(h \geq h_{\text {crit }} \mid \bar{h}_{\max }, \sigma_{h}\right)=0.40$, 地 震海啸发生率

$$
N_{l}\left(r, h_{\text {crit }}\right)=\int_{m_{\min }}^{m_{\max }} n_{l}(r, m) \phi\left(h \geq h_{c r i t} \mid \bar{h}_{\max }, \sigma_{h}\right) d m=0.20
$$

同理, 可得到马尼拉海沟和琉球海沟俯冲带对 应特定场点 1 的地震海啸发生率（表 8 ）。需 注意, 在计算特定场点的地震海啸总发生率 时, 要乘上逻辑树模型中各分支的系数。

表 8 中国东南沿海地区特定场点 1 的地震海 啸发生率

\begin{tabular}{ccc}
\hline & 马尼拉海沟 & 琉球海沟 \\
\hline 地震海啸发生率 & 0.14 & 0.26 \\
\hline
\end{tabular}

由于, 本文耦合的是震级上限的不确定性 效应, 其时间尺度趋于无穷大。因此, 中国东 南沿海地区特定场点在遭受震级上限时海啸 波高超过 $0.5 \mathrm{~m}$ 的地震海啸危险性为

$$
P\left(r, T, h_{c r i t}\right)=1-e^{-N\left(r, h_{c r i t}\right)}=0.33 \text { (3-2) }
$$

以此类推, 可得到中国东南沿海地区特定场点 的地震海啸危险性（表 9）。

表 9 中国东南沿海地区特定场点的地震海啸 危险性

\begin{tabular}{lccccc}
\hline & $\begin{array}{c}\text { 特定 } \\
\text { 场点 } 1\end{array}$ & $\begin{array}{c}\text { 特定 } \\
\text { 场点 } 2\end{array}$ & $\begin{array}{c}\text { 特定 } \\
\text { 场点 } 3\end{array}$ & $\begin{array}{c}\text { 特定 } \\
\text { 场点 } 4\end{array}$ & $\begin{array}{c}\text { 特定 } \\
\text { 场点 } 5\end{array}$ \\
\hline $\begin{array}{c}\text { 地震 } \\
\text { 海啸 }\end{array}$ & & & & & \\
危险 & 0.33 & 0.32 & 0.10 & 0.32 & 0.26 \\
性 & & & & & \\
\hline 5.结论 & & & & &
\end{tabular}

(1)本文探讨了耦合潜源区震级上限估计 值和震源深度不确定性效应的地震海啸危险 性分析方法, 并将其应用到中国东南沿海地区 特定场点的地震海啸危险性估计中。

(2)利用广义极值模型, 假定潜在地震海啸 
Risk Analysis and Crisis Response in Big Data Era (RAC-16)

源区震级上限的不确定性服从正态分布, 得马 尼拉海沟俯冲带不确定性特征为

$\mathrm{M} \sim \mathrm{N}(7.9,0.1)$; 琉球海沟俯冲带的不确定性特

征为 $\mathrm{M} \sim \mathrm{N}(8.5,0.7)$ 。

(3)假设特定场点遭受地震海啸灾害袭击 的事件为泊松过程。得到中国东南沿海地区五 个特定场点在遭受震级上限时海啸波高超过 $0.5 \mathrm{~m}$ 的地震海啸危险性分别为 $0.33,0.32,0.10,0.32,0.26$ 。

\section{Acknowledgements}

This study was supported by National Natural Science Foundation of China (No.41276020), and The Fundamental Research Funds for the Central Universities(No.ZY20160311), and The Fundamental Research Funds for the Central Universities(No.ZY20160312)

\section{致谢}

本研究得到了国家自然科学基金项目 (41276020) 和中央高校基本科研业务费 （ZY20160311）; 中央高校基本科研业务费 (ZY20160312) 的资助。

\section{参考文献}

[1]李伟,朱元清,王本龙,宋治平.海啸在冲绳海 槽和东海浅水大陆架地形上产生和传播 的数值模拟. 地震地磁观测与研 究,2007,28(5):138-142.

[2]温燕林,于海英,朱艾斓,宋治平,等.设定琉球 海沟发生罕遇地震评估我国东南沿海地 区的海啸风险.地震工程学报, 2016, 38(2):285-291

[3]张锟, 任鲁川, 田建伟, 刘哲.基于广义极 值理论的潜在地震海啸源震级上限估计 —以琉球海沟俯冲带为例. 第十七届中 国海洋 (岸) 工程学术讨论会论文 集,2015:1089-1094. [4]谢卓娟,吕悦军,兰景岩,徐丹丹.b 值和 V4 的 统计分析及其不确定性对地震危险性分 析结果的影响研究. 地震研究, 2013, 36(1):86-92.

[5]黄玮琼, 吴宣. 地震活动性参数不确定性对城 镇危险性估计的影响.地震学报, 2003, 25(6): 615-620

[6]漂家全, 韩炜, 高孟潭. 地震活动性参数的不 确定性及其对区划结果的影响.中国地震, 1996,12(增刊):71-77.

[7]高玉峰,谢康和,曾国熙,颜志平,等.地震危险 性概率分析中不确定性因素的研究.浙江 大学学报,1998,(5):590-597.

[8]王培涛,于福江,赵联大,侯京明.温州瓯江口 浅滩地区越洋海啸影响评估计算.海洋预 报,2013,30(4):18-26.

[9]毛献忠,祝倩,Wei Yong.浙江沿海潜在区域 地震海啸风险分析. 海洋学报, 2015, 37(3):37-45.

[10]温瑞智,任叶飞.中国地震海啸危险性分析 方法研究.世界地震工程,2007,23(1):6-11.

[11]任鲁川, 霍振香, 洪明理. 耦合潜源参数 不确定性效应的地震海啸危险性分析 一一原理与方法.海洋预报，2014，31(6): 7-13.

[12] E.L. Geist, T. Parsons. Probabilistic analysis of tsunami hazards. Nature Hazard, 2006, 37: $277-314$

[13]钱小仕,王福昌,曹桂荣,任晴晴.广义极值分 布在地震危险性分析中的应用.地震研 究,2012,35(1):73-78.

[14]J.P. Yan, S.S. Li， J. Bai， X.Y. Liu. The spatial symmetry axis of earthquake hazard in China. Journal of Risk Analysis and Cri sis Response,2013, 3(1): 59-64.

[15]X. M.Wang, Philip Liu. Preliminary study of possible tsunamis hazards in taiwan region,2006.http://ceeserver.cee.cornell.edu /pll-group/comcot.ht 